
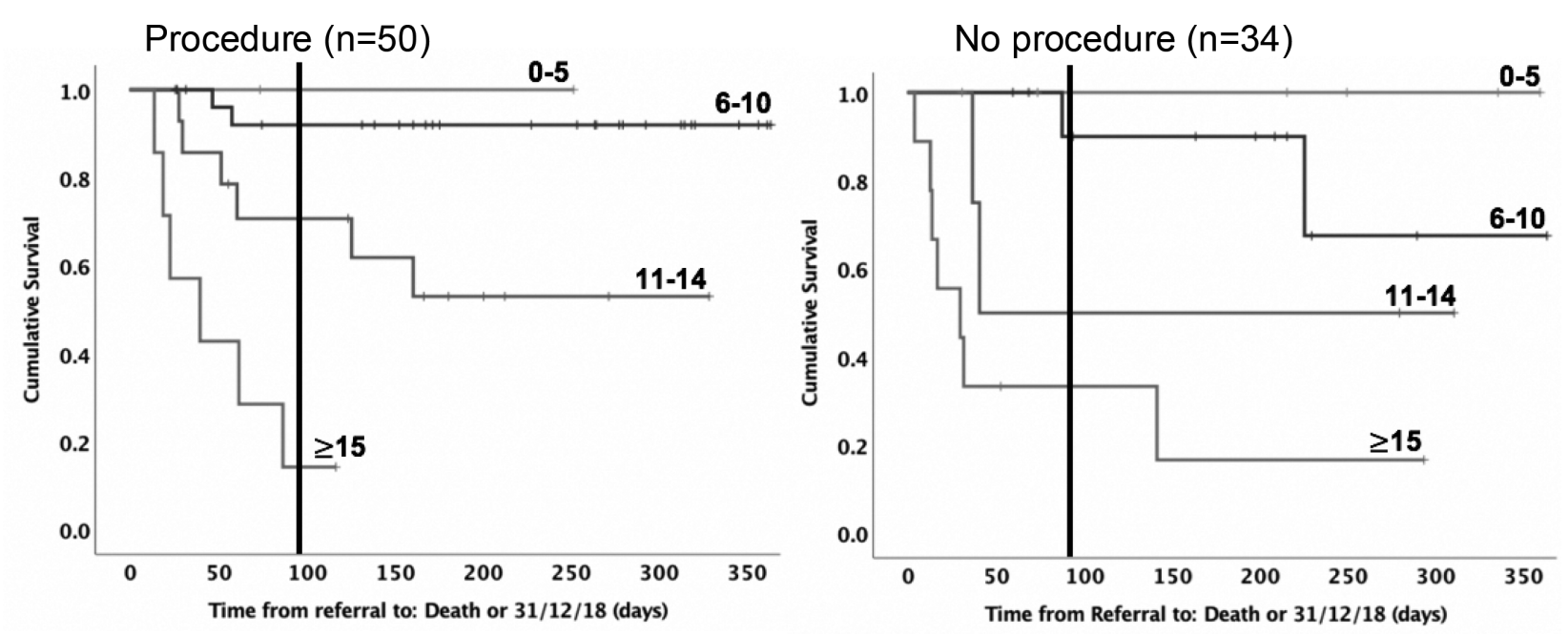

Abstract PWE-019 Figure 1 Cumulative survival in gastrostomy referrals, by procedural status

$\geq 15$ : high risk, unlikely to benefit. The aim of this one-year prospective study was to further evaluate the predictive validity of the RFH-GRS.

Methods All gastrostomy referrals from 1 January to 31 December 2018 were allocated a RFH-GRS score as part of their eligibility assessment. Patients were followed until death, or the end of the study period. Risk-benefit was assessed by comparing RFH-GRS and survival outcomes in patients who did and did not undergo the procedure.

Results Eighty-four patients were referred (58\% men; median [range] age, 72 [1-6] yr); dysphagia was the main indication in $71.4 \%$. Of the 84 referrals, 50 underwent placement, while 34 did not: too well $(n=11)$, too unwell $(n=14)$, declined $(n=3)$, unsuitable anatomy $(n=6)$. The median RFH-GRS was 10 (range -0 ). Patients with scores of -0 who underwent the procedure had a 93\% 90-day survival, compared with $89 \%$ in those who did not. However, 1-year survival rates were 93\% and $68 \%$ indicating clear long-term benefit (Figure 1). Patients with scores of 1-4 who underwent the procedure benefited in the short term with an $18 \%$ difference in 90-day survival (70\% vs $52 \%$ ) but 1 -year survival rates were similar at $55 \%$ and $50 \%$. Patients with scores $\geq 15$ who underwent the procedure had a significantly lower 90-day survival compared to those who did not (15\% vs 33\%).

Conclusion This prospective study provides further evidence to support the use of the RFH-GRS as a risk-benefit assessment tool in patients referred for gastrostomy placement.

\section{PWE-020 CATHETER-ASSOCIATED COMPLICATIONS INCLUDING DEEP VEIN THROMBOSIS IN A HPN COHORT: THE LEICESTER INTESTINAL FAILURE TEAM}

Syazeddy Samani ${ }^{\star}$, Karuna Kodali, Melanie Baker, Dan Rogers, James Stewart. University Hospitals of Leicester NHS Trust, Leicester, UK

\subsection{6/gutjnl-2019-BSGAbstracts.351}

Introduction Deep vein thrombosis (DVT) is a recognized complication associated with central catheters and it is thought that peripherally inserted central venous catheters (PICC) were associated with higher rates compared to centrally inserted catheters (CVC) but studies performed were not always in patients with home parenteral nutrition (HPN). ${ }^{1,2}$ The aim of our analysis is to assess the incidence of catheter-associated deep vein thrombosis (CA-DVT) and evaluate different rates of catheter-associated complication (CA-C) between the PICC and CVC group in a HPN cohort.

Method This is a retrospective, single centre cohort analysis of patients on HPN who had either PICC or CVC. The number of catheters removed due to CA-C from the 1st of January 2018 to 31st of December 2018 was reviewed and expressed in episodes. Data on CA-C which consisted of CA-DVT, confirmed and suspected catheter-associated infection (CA-I), catheter-associated device dysfunction (CA-DD) including line fracture and occlusion were analysed. Rates were calculated based upon the number of catheter and catheter re-inserted during this time period. Cumulative episodes and rates were compared between the two groups. Fisher's exact test was used for statistical analysis.

Results 64.3\% ( $\mathrm{n}=45)$ had CVC (Hickman lines) and 35.7\% $(n=25)$ had PICC with 25 episodes of catheter removal $(n=19$ and $n=9$ respectively, multiple episodes per patient allowed). The overall rate of CA-DVT was low at $1.4 \%(n=1)$ and this was associated with a PICC. There was no reported deep vein thrombosis within the CVC group. The cumulative episodes of CA-C appeared higher in the CVC group compared to the PICC group although the difference in rates was not statistically significant $(42.2 \% \mathrm{n}=19$ vs $36 \% \mathrm{n}=9, \mathrm{p}=$ 0.7994). There were 9 episodes of CA-I and 10 episodes of CA-DD in the CVC group compared to 3 episodes of CA-I and 5 episodes of CA-DD in the PICC group. Statistical analysis showed no difference in rates of CA-I $(p=0.5029)$ or CADD $(p=1)$ between the two groups.

Conclusions This analysis showed an overall low rate of catheter-associated deep vein thrombosis within a HPN cohort in a 12 month period. There was no significant difference in rates of catheter-associated complication between PICC and CVC.

\section{REFERENCES}

1. Bonizzoli M, Batacchi $S$, et al. Peripherally inserted central venous catheters and central venous catheters related thrombosis in post-critical patients. Intensive Care Med. 2011;37(2::284.

2. Vineet Chopra et al. Risk of venous thromboembolism associated with peripherally inserted central catheters: A systematic review and meta-analysis. The Lancet. 2013;vol 382 issue 9889; 31-25. 